\title{
So you want to conduct a randomised trial? Learnings from a 'failed' feasibility study of a Crisis Resource Management prompt during simulated paediatric resuscitation
}

\author{
Rachel Teis $^{\mathrm{a}, \mathrm{b}, \mathrm{c}}$, Jyai Allen ${ }^{\mathrm{a}, \mathrm{d}, *}$, Nigel Lee ${ }^{\mathrm{d}}$, Sue Kildea ${ }^{\mathrm{a}, \mathrm{d}, \mathrm{e}}$ \\ a Midwifery Research Unit, Mater Research Institute and University of Queensland, Mater Health, Raymond Terrace, South Brisbane, Queensland 4101, \\ Australia \\ b Paediatric Intensive Care Unit, Mater Children's Hospital, Mater Health, Raymond Terrace, South Brisbane, Queensland 4101, Australia \\ c School of Nursing, Midwifery and Paramedicine, Australian Catholic University, PO Box 456, Virginia, Queensland 4101, Australia \\ d School of Nursing, Midwifery and Social Work, Level 3, Chamberlain Building (35), University of Queensland, St Lucia, Queensland 4165, Australia \\ e Mothers, Babies and Women's Health, Mater Health, Raymond Terrace, South Brisbane, Queensland 4101, Australia
}

\section{A R T I C L E I N F O}

\section{Article history:}

Received 18 July 2016

Received in revised form 7 December 2016

Accepted 8 December 2016

\section{Keywords:}

Simulation training

Crisis resource management

Healthcare

Cardiopulmonary resuscitation

Paediatrics

Feasibility study

Randomised controlled trial

\begin{abstract}
A B S T R A C T
Background: No study has tested a Crisis Resource Management prompt on resuscitation performance. Methods: We conducted a feasibility, unblinded, parallel-group, randomised controlled trial at one Australian paediatric hospital (June-September 2014). Eligible participants were any doctor, nurse, or nurse manager who would normally be involved in a Medical Emergency Team simulation. The unit of block randomisation was one of six scenarios ( 3 control:3 intervention) with or without a verbal prompt. The primary outcomes tested the feasibility and utility of the intervention and data collection tools. The secondary outcomes measured resuscitation quality and team performance.

Results: Data were analysed from six resuscitation scenarios ( $\mathrm{n}=49$ participants); three control groups $(n=25)$ and three intervention groups $(n=24)$. The ability to measure all data items on the data collection tools was hindered by problems with the recording devices both in the mannequins and the video camera. Conclusions: For a pilot study, greater training for the prompt role and pre-briefing participants about assessment of their cardio-pulmonary resuscitation quality should be undertaken. Data could be analysed in real time with independent video analysis to validate findings. Two cameras would strengthen reliability of the methods.
\end{abstract}

(C) 2016 College of Emergency Nursing Australasia. Published by Elsevier Ltd. All rights reserved.

\section{Introduction}

While cardiac arrest in hospitalised paediatric patients is rare, the outcomes are often poor [1]. The survival to hospital discharge rate for these patients is approximately $40 \%$; while $10-30 \%$ have unfavourable neurological outcomes [2]. Using early warning tools for signs of clinical deterioration [3], medical emergency teams (METs) [4] and education to improve the efficacy of cardiopulmonary resuscitation (CPR) $[5,6]$ are essential for optimising outcomes [7].

The aim of simulation in healthcare is to replicate some or nearly all of the essential aspects of a clinical situation so that the scenario may be more readily understood and managed when it occurs

\footnotetext{
* Corresponding author at: Midwifery Research Unit, Mater Research Institute and University of Queensland, Mater Health, Raymond Terrace, South Brisbane, Queensland 4101, Australia.

E-mail address: jyai.allen@mater.uq.edu.au (J. Allen).
}

in clinical practice [8]. Hence simulated learning is essential for acquiring procedural resuscitation skills [9].

Crisis Resource Management (CRM) skills including communication, leadership, knowledge of environment, teamwork, anticipation and planning, attention allocation, workload distribution and use of cognitive aids are of core importance to the practice of emergency medicine [10]. An effective team leader is identifiable, clinically experienced, communicates effectively and delegates tasks $[11,12]$. While effective leadership is associated with improved team performance [11,13], poor leadership is associated with the inability to perform the critical interventions required to effectively resuscitate a patient $[11,14]$. Cognitive aids, such as resuscitation algorithms increase the performance of critical interventions (e.g. timely defibrillation) [15]; and may reduce errors [10]. Therefore, use of cognitive aids (rather than the availability of cognitive aids) is a key component of CRM.

Use of CRM skills is associated with improved resuscitation performance [16-19] in paediatric settings [20]. Over the last decade, CRM training has been incorporated into simulated learning envi- 
ronments [20,21]. While CRM training reduces no-flow time (NFT) (i.e. time without cardiac compressions during CPR) and increases team leader verbalisations (direct orders) [22]; resuscitation teams often deviate from the resuscitation protocol $[23,24]$. A recent study reported that CPR quality and adherence to the national resuscitation guidelines was poor among paediatric residents [25]. A contributing factor may be that retention of resucitation knowledge and skills may not persist beyond three [26] to 12 months [27], particularly when used rarely.

A verbal prompt read aloud to trigger the steps of the emergency management algorithm, compared to no verbal prompt, appears to improve the execution of critical actions [23]. A verbal prompt which incorporates prompts for CRM principles (e.g. who is the team leader), as well as steps of the resuscitation protocol (e.g. reading aloud the steps of the algorithm when needed), may improve team performance during real or simluated resuscitation. We found no published studies which tested a verbal CRM prompt and compared to having access to a written algorithm. Therefore we planned to test the feasibility of conducting such a study.

\section{Methods}

\section{Study design}

A feasibility, unblinded, parallel group randomised controlled trial (RCT) was used to answer the question: is it feasible to compare resuscitation performance in METs that use a verbal CRM prompt (intervention) compared to no verbal CRM prompt (control) through a RCT? The study was justified as the intervention had not been previously tested [28] and would enable investigation of the feasibility of the intervention and the data collection tools; for a larger trail that could assess the outcome of interest (i.e. resuscitation performance) $[29,30]$. Furthermore, feasibility trials allow for protocol modifications to be conducted prior to the conduct of a statistically powered RCT [31]. Ethical approvals from both the hospital (HREC/13/MHS/183) and the University (2014_155Q) were received.

\section{Research setting}

The study was conducted during June to September 2014 in a tertiary paediatric facility in Queensland, which admits over 15,000 children annually. When there is an unexpected clinical deterioration in a patient, within or outside the critical care areas, that meets MET criteria on the early warning tool; a MET code is called [32]. The composition of the MET is an ad-hoc combination of clinical hospital staff who attend an average of 120 calls for medical assistance each year. The MET emergency simulations run on a weekly basis throughout the study hospital.

\section{Participants}

The inclusion criterion for the study was any nurse, doctor, or ward-service staff employed at the paediatric tertiary hospital, who would normally be involved in MET events. Exclusions included refusal to participate or not receiving information and providing written informed consent prior to the simulation. Participants were not individually randomised.

\section{Recruitment}

The study was advertised on the Paediatric Resuscitation website on the hospital intranet. An email about the study including a link to information sessions was sent to relevant department managers to forward to staff. Participant information and consent forms were distributed at the information sessions and securely collected. Written informed consent remained valid for the entire study period. Efforts were made to collect individuals' written informed consent prior to simulation events. However, if this was not possible then clinicians were excluded from participating in the scenario. The bed managers, who were responsible for reading out the verbal prompt in the intervention groups; were briefed and consented separately.

\section{Randomisation and blinding}

The unit of randomisation was a paediatric resuscitation scenario with or without a verbal CRM prompt. The random allocation sequence for 24 scenarios using block randomisation (blocks of six) was generated by the hospital research facility. Block randomisation was used to ensure even distribution between the control and treatment arms of the study and reduce the chance that the order of randomisation could be predicted [33]. There was no way to blind participants or data analysers to the intervention.

\section{Interventions}

\section{Control}

Nursing staff were pre-briefed by the simulation coordinator and orientated to the Laerdal ${ }^{\circledR}$ Quality CPR (QCPR) infant manikin's features. The manikin provided key simulation functionalities including spontaneous breathing, electrocardiograph, live defibrillation, intravenous access, blood pressure and stores CPR information electronically. A scenario was provided and nursing staff were prompted to provide clinical care for the patient. At some point in the scenario, MET criteria were reached and staff activated the emergency buzzer. After the MET arrived, one of six simulation scenarios were given, all of which included a loss of cardiac output at four minutes $(+/-30 \mathrm{~s})$ after the activation of the emergency alarm. Both control and intervention arms had access to the resuscitation algorithm (Fig. 1). The manikin was pulseless for $8 \mathrm{~min}+/-30 \mathrm{~s}$ in each simulation. The simulation coordinator terminated the scenario after CPR data had been collected and the group attended a debrief session.

\section{Intervention}

The intervention scenario occurred in the same way as the control scenario with the addition of a Nurse Bed Manager using a stop watch and a schedule of timed prompts which focussed on team leadership and use of the resuscitation algorithm (two CRM principles)(Fig. 1).

\section{Outcomes}

\section{Primary outcomes (feasibility)}

- The number of simulations that are successfully conducted during the study period $(\mathrm{n})$;

- The ability to measure all data items during video analysis (yes/no).

\section{Secondary outcomes}

Resuscitation performance

- Proportion of CPR time with hands-on-chest (mean\%);

- Compression rate (mean\%);

- Compressions with correct hand position (mean\%);

- Adequate depth of compressions (mean\%);

- Compressions fully released (mean\%);

- Ventilation rate (mean\%); 


\begin{tabular}{|c|c|c|}
\hline \multicolumn{3}{|c|}{ Prompt Schedule - Intervention } \\
\hline When CPR Commences & $\begin{array}{l}\text { Start stopwatch } \\
\text { Correct CPR quality as needed } \\
\text { Rate } 100 \mathrm{bpm} \\
\text { Depth } 1 / 3 \text { chest wall } \\
\text { Swapping rescuers every } 2 \text { minutes } \\
\text { Minimal interruptions in CPR } \\
2 \text { minutes of CPR after return of spontaneous output (look for saturation trace) } \\
\end{array}$ & $\square$ \\
\hline At 1 minute post CPR & $\begin{array}{l}\text { Clearly state "Who is the Team Leader?" (If a conversation about who is TL has not started re-prompt } \\
\text { again). Do this until there is verbalisation of who team leader. } \\
\text { State clearly to the group their name, that they are the team leader and to follow their direction. } \\
\text { If you do not know their name, ask then tell the group as above. }\end{array}$ & $\square$ \\
\hline At 2 minutes post CPR & $\begin{array}{l}\text { If no one is using the cardiac arrest algorithm (reading it out for the team to follow) - clearly state to } \\
\text { the TL; } \\
\text { "I can read the arrest protocol for you to follow. Is this a shockable or non-shockable rhythm?" } \\
\text { Repeat this step until a team leader verbalises the rhythm. } \\
\text { After the non-shockable or shockable rhythm is verbalised by the TL, read out the interventions } \\
\text { required. } \\
\text { Continue to do so until requested otherwise or on return of output. }\end{array}$ & $\square$ \\
\hline $\begin{array}{l}\text { After the first dose of } \\
\text { adrenaline }\end{array}$ & $\begin{array}{l}\text { Prompt the TL - Clearly state; } \\
\text { "Please consider the } 4 \mathrm{H}^{\prime} \mathrm{s} \text { and T's to reverse the arrest". } \\
\text { Share the cardiac arrest algorithm with the TL and point to the } 4 \mathrm{H} \text { 's and T's. } \\
\text { Do this every } 5 \text { minutes until diagnosis or exclusion of diagnosis is established. }\end{array}$ & $\begin{array}{l}\square \\
\square\end{array}$ \\
\hline
\end{tabular}

Fig. 1. Prompt schedule (intervention).

- Administration of adrenaline every $4 \mathrm{~min}+/-30 \mathrm{~s}$ (measured from the initial dose) (\% n).

\section{Team performance}

- The proportion of team members that identified a clearly allocated team leader (\% n);

- Difference in the teamwork observed at the simulation as measured by the scale on the TEAM tool [34] (mean score);

Sample size

The sample size for the feasibility study was a pragmatic calculation estimating that 24 simulations could be conducted within the study period. The feasibility study was not powered to detect significant differences on clinical measures.

\section{Data collection}

Data were collected from three primary sources: video recording of MET simulations, quality CPR (QCPR) data and participant surveys.

Video recordings of the team scenarios were used to evaluation teamwork behaviours using the TEAM tool (Fig. 2) and to provide data of adherence to resuscitation guidelines using a data collection tool (Fig. 3). The TEAM tool uses 11 assessment items (0-4 scale) to measures leadership, communication, cooperation and coordination, team climate, adaptability, situational awareness, prioritisation and clinical standards; the final assessment item provides an overall 'global' rating of team performance (0-10 scale); total scores range for 0-54 [34]. The TEAM tool has recently been validated for use in simulated and real clinical settings [34].

The QCPR infant manikin has an internal data-recording device for measuring the quality of CPR using a variety of measurements (see Secondary Outcomes). These data were downloaded and analysed using Laerdal ${ }^{\circledR}$ Session Viewer software (Version 5.2.5821).

The authors developed a one-question paper survey which asked participants to circle 'yes' or 'no' in response to the question: "Did you feel you had a clearly identified team leader in the scenario?".

\section{Data analysis}

Two independent assessors, both clinical nurse educators with expertise in paediatric resuscitation, reviewed the video data and assigned a score using the TEAM tool. Consensus between the assessors was reached to determine a total score for each scenario and to determine which data items could and could not be collected.

\section{Statistical analysis}

Simple descriptive statistical methods measured the outcomes. 


\section{Team Emergency Assessment Measure (7\& $\mathbb{R}$ )}

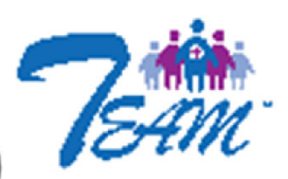

\section{Introduction}

This non- technical skills questionnaire has been designed as an observational rating score for valid, reliable and feasible ratings of emergency medical teams (e.g. resuscitation and trauma teams). The questionnaire should be completed by expert clinicians to enable accurate performance rating and feedback of leadership, team work, situation awareness and task management. Rating prompts are included where applicable. The following scale should be used for each rating:

\begin{tabular}{|c|c|c|c|c|}
\hline Never/Hardly ever & seldom & About as often as not & Often & Always/Nearly always \\
\hline 0 & 1 & 2 & 3 & 4 \\
\hline
\end{tabular}

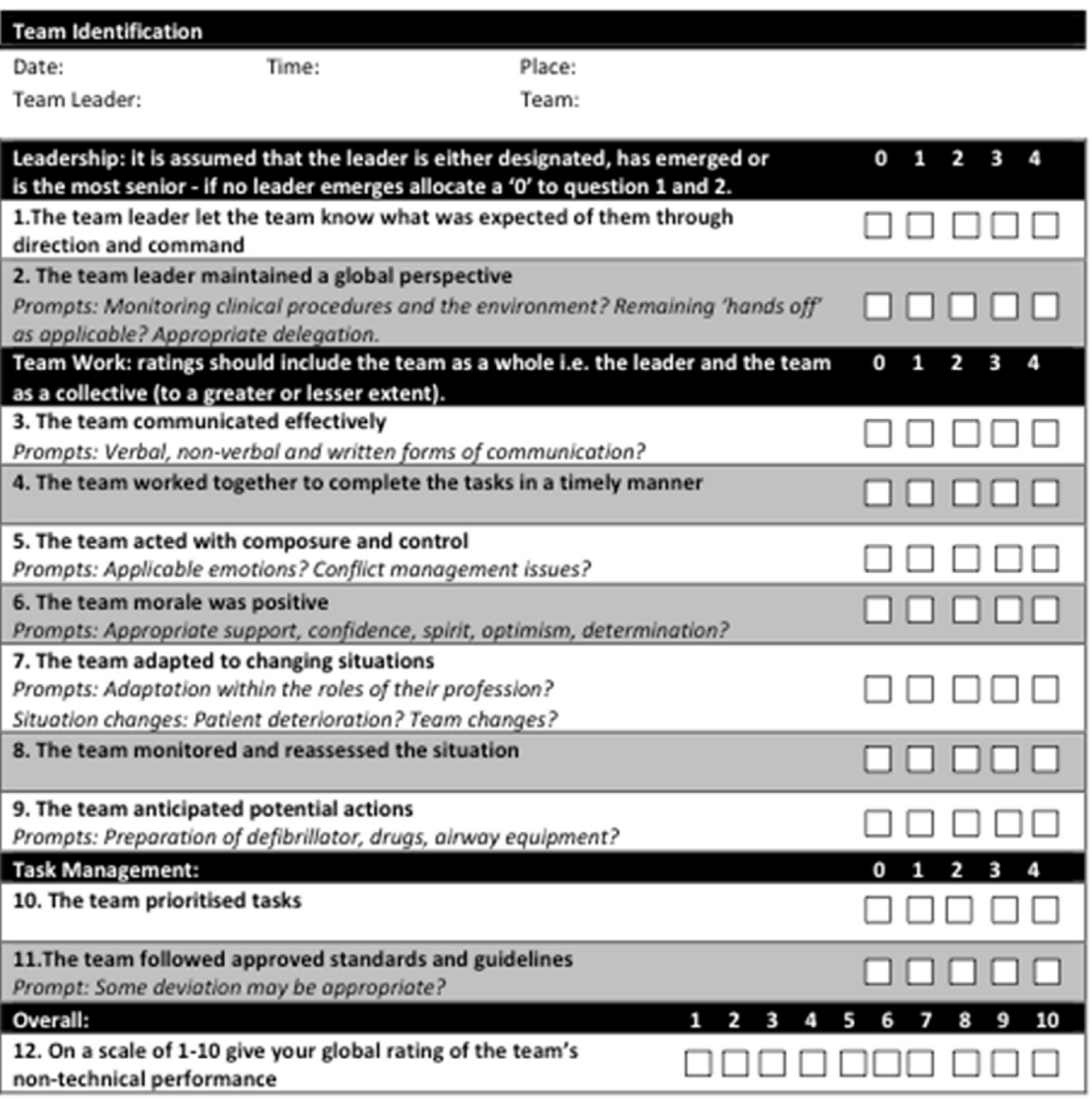

Comments: 


\section{Mater}

Medical Research Institute

\section{CueME Study Data Collection Tool} $+$

Simulation Number:

Control $\square$

Intervention $\square$

\begin{tabular}{|c|c|}
\hline CPR & Times recorded \\
\hline Time CPR was commenced & $\longrightarrow:$ \\
\hline Hands on chest time (from Wireless Skill Reporter) & $-{ }^{\prime}$ \\
\hline \multirow{5}{*}{$\begin{array}{l}\text { What was the nature of the CPR quality prompt? } \\
\text { - Rate } 100 \mathrm{bpm} \\
\text { - } \text { Depth } 1 / 3 \text { chest wall } \\
\text { - Swapping rescuers every } 2 \text { minutes } \\
\text { - Minimal interruptions in CPR }\end{array}$} & \\
\hline & \\
\hline & \\
\hline & \\
\hline & \\
\hline Adrenaline & Response \\
\hline Was adrenaline given in the scenario? & $\begin{array}{l}\text { Please tick } \\
\text { Yes } \square \quad \text { No } \square\end{array}$ \\
\hline \multicolumn{2}{|l|}{ Time of initial adrenaline dose } \\
\hline Time of second adrenaline dose & 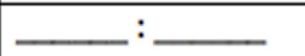 \\
\hline Team Leader Involvement & Times recorded \\
\hline \multicolumn{2}{|l|}{$\begin{array}{l}\text { Number of people who felt that there was a clearly allocated } \\
\text { team leader in the scenario? }\end{array}$} \\
\hline \multicolumn{2}{|l|}{$\begin{array}{l}\text { Number of people who did not feel there was a clearly allocated } \\
\text { team leader in the scenario? }\end{array}$} \\
\hline Prompting & Response \\
\hline \multicolumn{2}{|l|}{$\begin{array}{l}\text { The number of times that the prompt failed to follow the prompt } \\
\text { schedule }\end{array}$} \\
\hline Resuscitation & Response \\
\hline $\begin{array}{l}\text { Did any other person in the simulation read the resuscitation } \\
\text { algorithm at any other time? }\end{array}$ & $\begin{array}{l}\text { Please tick } \\
\text { Yes } \square \quad \text { No } \square\end{array}$ \\
\hline $\begin{array}{l}\text { Did the prompt read aloud the resuscitation algorithm in the } \\
\text { scenario? }\end{array}$ & $\begin{array}{l}\text { Please tick } \\
\text { Yes } \square \quad \text { No } \square\end{array}$ \\
\hline Cause of Arrest & Time \\
\hline Time taken to verbalise the cause of arrest from the onset of CPR & $\longrightarrow:$ \\
\hline
\end{tabular}

Fig. 3. Data Collection Tool (video analysis). 


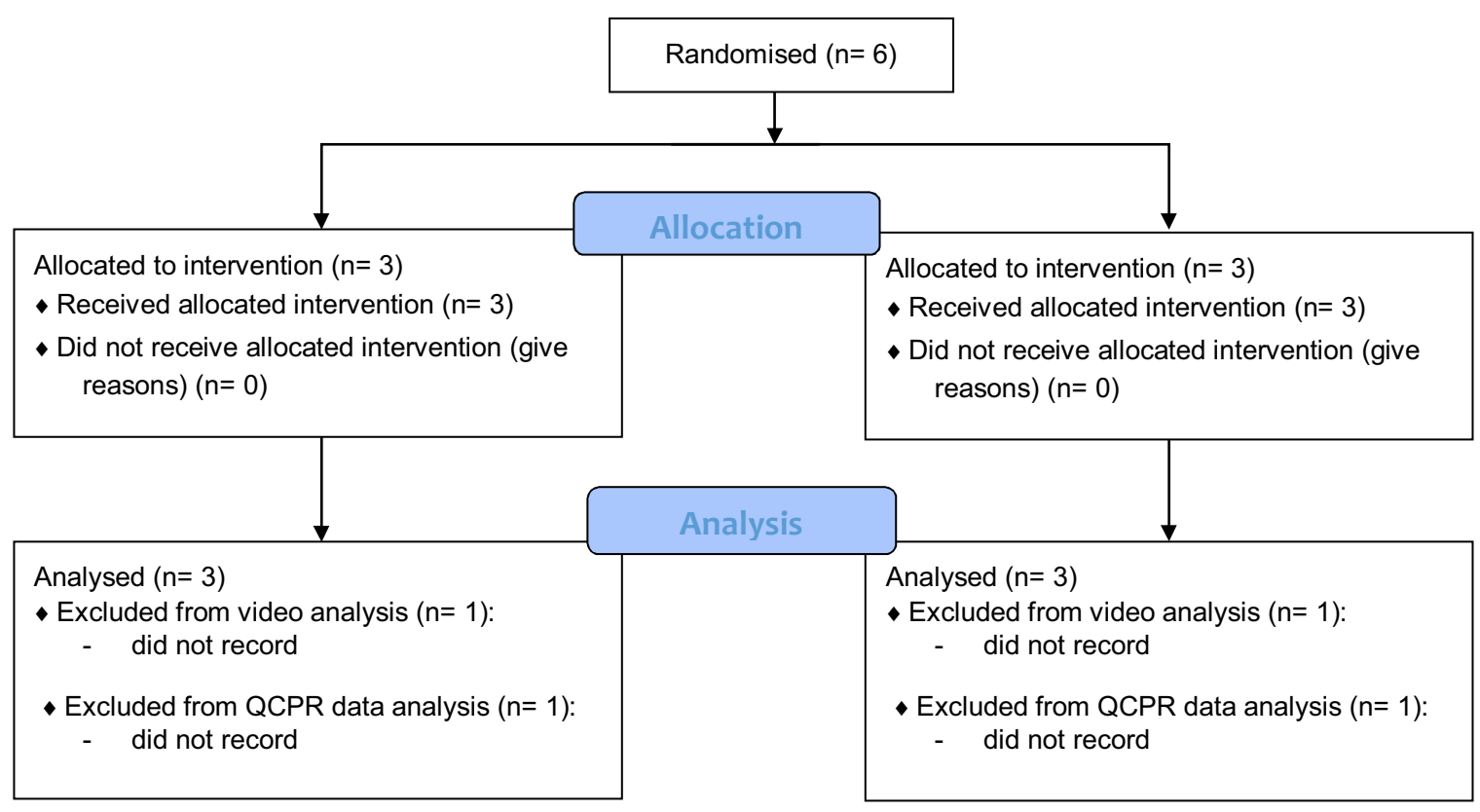

Fig. 4. Participant Flow Diagram.

\section{Results}

\section{Feasibility outcomes}

\section{Recruitment}

We anticipated 24 simulations would be randomised and run during the study period. The closure of the study hospital, however, led to cancellation of some of the timetabled simulations due to other priorities such that only six simulations were able to be conducted (see Fig. 4). A total of 49 staff participated in the trial with no declines or withdrawals.

\section{Data collection}

Of the six simulations, data collection issues resulted in video recording for four scenarios (66\%), QCPR data for four scenarios (66\%), and participant feedback from six scenarios (100\%). There were several problems with video data collection. On two occasions the video failed to record which meant that teamwork scores and some resuscitation performance measures (e.g. timing of adrenaline) were unable to be analysed. There were frequent problems with poor quality audio or picture, including one instance where the video camera detached from the wall and another where it was accidentally moved during the scenario such that the resuscitation area was no longer in camera range. Aforementioned missing data, or problems with video data quality (e.g. camera out of frame), meant it was not feasible to measure all data items on the data collection tool (Fig. 3). Specifically 1 ) the number of times the resuscitation algorithm was read by the prompt or any other person, 2 ) the number of times the prompt schedule was correctly/incorrectly used, 3) the number of times the team followed the prompt aloud, 4) the length of time for the team to verbally identify the cause of the arrest, and 5) the number of factors that affected ease or difficult of prompt execution.

\section{Secondary outcomes}

\section{Resuscitation outcomes}

The intervention groups had a higher proportion of CPR time with hands-on-chest (81\%) compared to the control groups (69\%). Compression rates were in excess of the recommended 100 per minute (128-157 per minute); inadequate hand position occurred more than $50 \%$ of the time; inadequate depth of chest compression occurred more than $70 \%$ of the time and inadequate release of chest compression occurred more than $35 \%$ of the time. Adrenaline was not consistently administered every four minutes $(+/-30 \mathrm{~s})$ in any scenario. Timing ranged from 2:30-5:41 in the intervention groups compared to 2:38-3:15 in the control groups.

\section{Teamwork outcomes}

The proportion of team members who identified a team leader was higher in the control group $(87 \%, \mathrm{n}=25)$ compared to the intervention group $(72 \%, \mathrm{n}=24)$. The mean TEAM score was higher in the control group (51) compared to the intervention group (42). These results are based on four simulations only.

\section{Discussion}

This is the first study to assess the feasibility of testing a verbal CRM prompt to improve resuscitation performance. The strength of a feasibility RCT is the ability to identify methodological and technical issues which can be redesigned and controlled for in a main study; and to provide preliminary data which can be used to calculate a sample size [30]. The findings indicate that significant protocol modifications are required to render the study design feasible for a full-scale trial.

While only six scenarios were able to be conducted during the study period; it was large enough to provide information about the intervention prompt and data collection that allows modification for a full-scale trial.

\section{Protocol fidelity}

In this study the intervention, verbal CRM prompt, was performed by a different person each time. While this replicated a real life scenario it threatened trial fidelity as the verbal prompts could be ineffective. For example, the prompt schedule (Fig. 1) advised the bed manager, (the prompt) to "correct CPR quality as needed", however this was rarely witnessed on the video footage. More comprehensive training and practice of the prompt role prior to a future 
study may assist with ensuring protocol fidelity; which increases the chances of a measurable effect.

\section{Data collection and analysis}

As highlighted previously, video equipment and audio-visual quality issues contributed to problems with data collection. Without adequate footage it was difficult to analyse how and when the verbal prompts were performed, and when the team was following a prompt. This would be easier to manage in a simulation laboratory however the simulations are conducted in various locations throughout the hospital to emulate realistic clinical conditions. Alternatively a second camera would provide a back-up in those situations where initial footage was not usable. Real time analysis of the scenario by a clinical expert not directly involved in the research might assist in recording all items on the TEAM tool (Fig. 2) and data collection tool (Fig. 3). Independent video analysis by a different clinical expert could be used to validate the measures on the aforementioned tools.

\section{CPR performance}

Effective simulation requires deliberate practice, feedback on performance and high-stakes testing [35]. In this feasibility trial the results depicted lower than expected CPR performance in both groups. Some studies have reported significant variation in CPR practice compared to current paediatric CPR standards despite targeted training regimes and visual feedback from video recorded simulations [36], whilst others have reported compliance in rate but variation in depth and release [37]. Nevertheless, we interpret that participants did not perform simulated CPR akin to the standard they would use in a real life scenario. In a future study, it might assist to implement strategies that increase the quality of participants' CPR performance by increasing the stakes (for example pre-briefing participants that the quality of their CPR performance will be measured and assessed during simulations). In any case, if the results of a future trial demonstrated the intervention was effective during simulated CPR; the generalisability of the results to real CPR events would benefit from testing in the clinical setting using real scenarios.

\section{Conclusions}

Application of CRM principles, simulated emergency drills, and the use of a verbal prompt during emergency management; all improve resuscitation performance as stand-alone interventions. In order to trial the use of a verbal CRM prompt during simulated resuscitation scenarios: 1 ) greater training for the prompt role would need to be undertaken, 2) participants should be pre-briefed that their CPR quality is being measured, and 3) data could be collected in real time and independently assessed using video data for validation.

\section{Authorship statement}

RT and SK conceived and designed the study. RT secured funding. RT, NL and SK developed the study protocol. RT undertook data collection under the supervision of SK. JA analysed and interpreted the data. RT, JA, NL, and SK prepared and approved the manuscript.

\section{Role of the funding source}

This work was supported by a Mater Children's Hospital Golden Casket Grant [ grant number 2252, 2013] awarded to the first author and disbursed through Mater Research. The funding body had no role in the design of the study, data collection, analysis, interpretation, nor writing of the manuscript.

\section{Conflicts of interest}

The authors declare no conflicts of interest.

\section{Acknowledgements}

The authors wish to acknowledge Andrew Heasley (Simulation Co-ordinator) who facilitated the simulations and assisted with independent analysis of the video data.

\section{References}

[1] Nadkarni VM, Larkin GL, Peberdy MA, Carey SM, Kaye W, Macini ME, et al. First documented rhythm and clinical outcome from in-hospital cardiac arrest among children and adults. JAMA 2006;295(1):50-7.

[2] Girotra S, Spertus JA, Li Y, Berg RA, Nadkarni VM, Chan PS. Survival trends in pediatric in-hospital cardiac arrests: an analysis from Get With the Guidelines-Resuscitation. Circ Cardiovasc Qual Outcomes 2013;6(1):42-9.

[3] Haines C, Perrott M, Weir P. Promoting care for acutely ill children-development and evaluation of a paediatric early warning tool Intensive Crit Care Nurs 2006;22(2):73-81.

[4] Jones DA, DeVita MA, Bellomo R. Rapid response teams. N Engl J Med 2011:365(2):139-46.

[5] Hunziker S, Bühlmann C, Tschan F, Balestra G, Legeret C, Schumacher C, et al. Brief leadership instructions improve cardiopulmonary resuscitation in a high-fidelity simulation: a randomized controlled trial. J Crit Care Med 2010;38(4):1086-91.

[6] Hunziker S, Johansson AC, Tschan F, Semmer NK, Rock L, Howell MD, et al. Teamwork and leadership in cardiopulmonary resuscitation. J Am Coll Cardiol 2011:57(24):2381-8.

[7] Niles DE, Dewan M, Zebuhr C, Wolfe H, Bonafide CP, Sutton RM, et al. A pragmatic checklist to identify pediatric ICU patients at risk for cardiac arrest or code bell activation. Resuscitation 2016:99:33-7.

[8] Hovancsek M. Using simulation in nurse education, Simulation in Nursing Education: from Conceptualization to Evaluation. In: Jeffries PR, editor. New York: National League for Nursing; 2007. p. 1-9.

[9] Nolan JP, Soar J, Zideman DA, Biarent D, Bossaert LL, Deakin C, et al. European resuscitation council guidelines for resuscitation 2010 Section 1. Executive summary. Resuscitation 2010;81:1219-76.

[10] Carne B, Kennedy M, Gray T. Review article: crisis resource management in emergency medicine. Emerg Med Aust 2012;24(1):7-13.

[11] Fernandez CE, Russo SG, Riethmüller M, Boos M. Effects of team coordination during cardiopulmonary resuscitation: a systematic review of the literature. J Crit Care 2013;28(4):504-21.

[12] Andersen PO, Jensen MK, Lippert A, Ostergaard D, Klausen TW. Development of a formative assessment tool for measurement of performance in multi-professional resuscitation teams. Resuscitation 2010;81:703-11.

[13] Burke CS, Stagl KC, Klein C, Goodwin GF, Salas E, Halpin SM. What type of leadership behaviors are functional in teams? A meta-analysis. Leadersh Q 2006; 17:288-307.

[14] Marsch SCU, Müller C, Marquardt K, Conrad G, Tschan F, Hunziker PR. Human factors affect the quality of cardiopulmonary resuscitation in simulated cardiac arrests. Resuscitation 2004;60:51-6.

[15] Nolan JP, Hazinski MF, Billi JE, Boettiger BW, Bossaert L, de Caen AR, et al. Part 1: executive summary: 2010 international consensus on cardiopulmonary resuscitation and emergency cardiovascular care science with treatment recommendations. Resuscitation 2010;81:e1-25.

[16] Yeung JHY, Ong GJ, Davies RP, Gao F, Perkins GD. Factors affecting team leadership skills and their relationship with quality of cardiopulmonary resuscitation. Crit Care Med 2012;40(9):2617-21.

[17] Chakraborti C, Boonyasai RT, Wright SM, Kern DE. A systematic review of teamwork training interventions in medical student and resident education. J Gen Intern Med 2008;23(6):846-53.

[18] Hunziker S, Tschan F, Semmer N, Howell M, Marsch S. Human factors in resuscitation: lessons learned from simulator studies. J Emerg Trauma Shock 2010;3(4):389-94.

[19] Manser T. Teamwork and patient safety in dynamic domains of healthcare: a review of the literature. Acta Anaesthesiol Scand 2009;53(2):143-51.

[20] Cheng A, Donoghue A, Gilfoyle E, Eppich WJ. Simulation based CRM training for pediatric critical care medicine a review for instructors. Pediatr Crit Care Med 2012;13(2):197-203.

[21] Gilfoyle E, Gottesman R, Razack S. Development of a leadership skills workshop in paediatric advanced resuscitation. Med Teach 2007;29(9):276-83.

[22] Fernandez Castelao E, Russo SG, Cremer S, Strack M, Kaminski L, Eich C, et al Positive impact of crisis resource management training on no-flow time and team member verbalisations during simulated cardiopulmonary resuscitation: a randomised controlled trial. Resuscitation 2011;82:1338-43. 
[23] Burden AR, Carr ZJ, Staman GW, Littman JJ, Torjman MC. Does every code need a reader? Improvement of rare event management with a cognitive aid "reader" during a simulated emergency: a pilot study. Simul Healthc 2012;7(1):1-9.

[24] Abella BS, Alvarado JP, Myklebust H, Edelson DP, Barry A, O’Hearn N. Vanden Hoek TL, Becker LB. Quality of cardiopulmonary resuscitation during in-hospital cardiac arrest. JAMA 2005;293(3):305-10.

[25] Hunt EA, Vera K, Diener-West M, Haggerty JA, Nelson KL, Shaffner DH, et al. Delays and errors in cardiopulmonary resuscitation and defibrillation by pediatric residents during simulated cardiopulmonary arrests. Resuscitation 2009;80:819-25.

[26] Mosley C, Dewhurst C, Molloy S, Shaw BN. What is the impact of structured resuscitation training on healthcare practitioners, their clients and the wider service? A BEME systematic review: BEME Guide No. 20. Med Teach 2012;34(6):e349-85.

[27] Lo BM, Devine AS, Evans DP, Byars DV, Lamm OY, Lee RJ, et al. Comparison of traditional versus high-fidelity simulation in the retention of ACLS knowledge. Resuscitation 2011;82:1440-3.

[28] Hertzog MA. Considerations in determining sample size for pilot studies. Res Nurs Health 2008;31:180-91

[29] Arain M, Campbell M, Cooper C, Lancaster G. What is a pilot or feasibility study? A review of current practice and editorial policy. BMC Med Res Methodol 2010;10:67.
[30] Thabane L, Ma J, Chu R, Cheng J, Ismaila A, Rios LP, et al. A tutorial on pilot studies: the what, why and how. BMC Med Res Methodol 2010;10:1.

[31] Bowen DJ, Kreuter M, Spring B, Cofta-Woerpel L, Linnan L, Weiner D, et al. How we design feasibility studies. Am J Prev Med 2009;36(5):452-7.

[32] Devita MA, Bellomo R, Hillman K, Kellum J, Rotondi A, Teres D, et al. Findings of the first consensus conference on medical emergency teams. Crit Care Med 2006;34(9):2463-78.

[33] Wang D, Bakhai A. Clinical trials: a practical guide to design, analysis, and reporting. London: Remedica; 2006.

[34] Cooper S, Cant R, Connell C, Sims L, Porter JE, Symmons M, et al. Measuring teamwork performance: validity testing of the Team Emergency Assessment Measure (TEAM) with clinical resuscitation teams. Resuscitation 2016;101:97-101.

[35] Steadman RH, Huang YM. Simulation for quality assurance in training, credentialing and maintenance of certification. Best Pract Res Clin Anaesthesiol 2012;26(1):3-15.

[36] Cheng A, Hunt EA, Grant D, Lin Y, Grant V, Duff JP, et al. Variability in quality of chest compressions provided during simulated cardiac arrest across nine pediatric institutions. Resuscitation 2015;97:13-9.

[37] Hsieh TC, Wolfe H, Sutton R, Myers S, Nadkarni V, Donoghue A. A comparison of video review and feedback device measurement of chest compressions quality during pediatric cardiopulmonary resuscitation. Resuscitation 2015;93:35-9. 